\title{
Fertilización en la producción de bulbos y flor de amancae (Ismene amancaes), bajo condiciones de invernadero
}

\author{
Fertilization on bulbs and flower production of amancae \\ (Ismene amancaes) under greenhouse conditions \\ Juan Carlos Jaulis C. ${ }^{*}$, Alejandro Pacheco A. ${ }^{2}$ y Ana Martinez V. ${ }^{3}$ \\ * Autor de correspondencia
}

\begin{abstract}
Resumen
El objetivo de la investigación fue determinar el efecto de la fertilización sobre la producción de bulbos y flor de amancae. Las variables evaluadas fueron crecimiento (altura, número de hojas, número de raíces y díametro de tallo por planta) y rendimiento (número de flores, días a la floración, diámetro y peso de bulbo final). El experimento se condujo bajo condiciones de invernadero y se utilizó 216 bulbos. Los tratamientos fueron diferentes dosis de fertilización de Nitrógeno (N), Fósforo (P) y Potasio (K) expresados en mg/kg: T1: 0-0-0, T2: 120-0-0, T3: 0-50-0, T4: 0-0-160, T5: 120-50-0, T6: 120-0-160, T7: 0-50-160 y T8: 120-50-160. Las fuentes utilizadas fueron, nitrato de amonio, superfosfato triple y cloruro de potasio. Los fertilizantes fosforados y potásicos fueron aplicados al inicio de la siembra, mientras las aplicaciones de nitrógeno se efectuaron a los 15, 45 y 75 días. Se utilizó un diseño completamente al azar (DCA), con ocho tratamientos y tres repeticiones, para la comparación de medias se utilizó la prueba de Duncan a un nivel de significancia de 0,05 . Los tratamientos 1, 3 y 4 registraron los mejores resultados para la variable crecimiento. Así mismo, el $\mathrm{T}_{4}$ presentó el mejor resultado para las variables de rendimiento, con 1,41 flores, 04,20 cm de diámetro final de bulbo y 40,89 g de peso final del bulbo. El $\mathrm{T}_{8}$ alargó el inicio de floración a 149,3 días. En conclusión la fertilización con NPK no incrementa el rendimiento de las variables de crecimiento, Sin embargo tienen un efecto positivo en las variables de rendimiento.
\end{abstract}

Palabras clave: crecimiento; rendimiento; fertilización; Ismene amancaes.

\begin{abstract}
The objective of the research was determine the effect of fertilization on the production of bulbs and amancae flower. The variables evaluated were growth (height, number of leaves, number of roots and diameter of stem per plant) and yield (number of flowers, days to flowering, diameter and final bulb weight). The experiment was conducted under greenhouse conditions and 216 bulbs were used. The treatments were different doses of fertilization of Nitrogen $(\mathrm{N})$, Phosphorus (P) and Potassium (K) expressed in mg / kg: T1: 0-0-0, T2: 120-0-0, T3: 0-50- 0, T4: 0-0-160, T5: 120-50-0, T6: 120-0-160, T7: 0-50-160 and T8: 120-50-160. The sources used were ammonium nitrate, triple superphosphate and potassium chloride. The phosphorus and potassium fertilizers were applied at the beginning of the sowing, while nitrogen applications were made at 15, 45 and 75 days. A completely randomized design (DCA) was used, with eight treatments and three repetitions, for the comparison of means the Duncan test was used at a significance level of 0,05 . Treatments 1 , 3 and 4 recorded the best results for the growth variable. Likewise, the T4 presented the best result for the performance variables, with 1,41 flowers, $04,20 \mathrm{~cm}$ of final diameter of bulb and 40,89 $\mathrm{g}$ of final weight of the bulb. T8 lengthened the flowering start to 149,3 days. In conclusion, fertilization with NPK does not increase the yield of the growth variables. However, they have a positive effect on the yield variables.
\end{abstract}

Keywords: increase; performance; fertilization; Ismene amancaes.

\section{Introducción}

La flor de amancae ha sido muy apreciada desde el pasado debido a su belleza (Pascual, 2007). Durante la fiesta de San Juan, en el mes de junio, la aristocracia limeña y el pueblo visitaban las Pampas de Amancaes, ubicadas al pie de las Lomas de Amancaes, en el distrito del Rímac (La República, 2017) Actualmente no queda rastro de lo que fue el hábitat natural de esta flor, que en estación de invierno florecía como un manto amarillo adornando la ciudad de Lima (Pascual, 2007).

En el Perú, se reconocen 24 géneros y 138 especies de la familia Amarilidaceae (Ulloa et al., 2004), de las cuales 54 son endémicas. Una de ellas es el I. amancaes, planta herbácea, de bulbo perenne (Pascual, 2007), emerge en el 
mes de junio, durante el invierno, con el inicio del período de neblina, luego presenta un desarrollo foliar, seguido de la floración, fructificación, producción de semillas y germinación hasta desarrollar el bulbo, que ocurre al terminar el periodo de humedad (Suni et al., 2011). Esta planta florece una vez al año, con un período de vida de dos a cuatro días (SERFOR, 2015). Sus flores son grandes, de color amarillo, con agradable aroma, muy apreciadas y de valor ornamental (Soto y Leiva, 2015). Es considerada la flor emblemática de la ciudad de Lima (Palacios, 2017). $\mathrm{Su}$ único medio de propagación natural es por semilla (sexual), pero también se puede propagar a través de los bulbos (asexual), llega a producir hasta 13 semillas por flor y siete flores por planta (dependiendo del tamaño del bulbo). Según el reporte de Agüero \& Suni (1999), el fenómeno del Niño afectó negativamente el proceso de floración, solo el $30 \%$ de las plantas florecieron bajo dichas condiciones. Además, el incremento urbano y el pastoreo de ganado han reducido drásticamente el área de distribución del I. amancaes en las lomas y el número de individuos en su medio ambiente natural, lo que ha llevado a considerarla una especie vulnerable (León et al. 2006).

El I. amancaes, es endémico del Perú, principalmente de las lomas de la costa central peruana (Agüero, 2002), Se encuentra en las lomas de Lúcumo, Pachacámac, VillaMaría y Paloma, ubicados en los distritos de Pachacamac, VillaMaría y Chilca, departamento de Lima, Perú, en el flanco occidental y partes bajas de las lomas, su hábitat es el fondo de las quebradas y laderas rocosas, donde la materia orgánica y la humedad son propicia para su desarrollo; generalmente se encuentran en grupos, conformados por más de un centenar de plantas. Se pueden encontrar hasta sobre los 600 m.s.n.m., ingresando a laderas altas de loma herbácea (SERFOR, 2015).

Por otra parte, la fertilización de los cultivos, es una herramienta agronómica que consiste en nutrir cada cultivo en forma óptima, con la mínima adición de fertilizante, de manera que sea económica para los agricultores y se minimice la contaminación (Mollenhauer, 2002). De acuerdo con Magalhães (1993), un criterio adecuado de fertilización, satisface las necesidades del cultivo mediante la adopción de técnicas que permiten el óptimo aprovechamiento de fertilizantes, agua, mano de obra y del resto de insumos, minimizando las pérdidas de nutrientes por lixiviación, erosión y volatilización. Según LópezMtz et al. (2001) la estructura del suelo es el principal factor que condiciona la fertilidad y productividad de los suelos agrícolas. Siendo esta característica física del suelo determinante para el establecimiento de un plan de fertilización en el cultivo de plantas bulbosas.

En relación al nitrógeno, León (2002) ha señalado que se desconoce la dosis adecuada de fertilización de determinadas especies bulbosas de flor. Sin embargo, se ha comprobado que a mayor dosis de nitrógeno, existe una mayor influencia sobre algunos parámetros como el incremento del largo de vara floral. Pinochet (1999) reportó que la demanda de nitrógeno para producir flores generalmente no es cubierta en su totalidad por el órgano de reserva, por tanto, la fertilización nitrogenada es necesaria para compensar el déficit de $\mathrm{N}$ en la producción de flores y follaje. También, determinó que la demanda de $\mathrm{N}$ para un rendimiento alto y medio es de $28 \mathrm{~g}$ de $\mathrm{N}^{*} \mathrm{~m}^{2}$ y $17 \mathrm{~g}$ de N.m ${ }^{-2}$ respectivamente.

Para Pinochet (1999) la sobre fertilización con P (niveles por sobre $50 \mathrm{mg} * \mathrm{~kg}^{-1}$ ) induce la deficiencia de zinc $(\mathrm{Zn})$, particularmente en suelos con niveles marginales de este elemento. Además, señala que el nivel crítico de $\mathrm{P}$ disponible, para un rendimiento alto esperado en plantas geófitas en general, es de $14 \mathrm{mg} \mathrm{P}^{*} \mathrm{~kg}^{-1}$ de suelo y el nivel crítico de $\mathrm{K}$ es de $140 \mathrm{mg} \mathrm{K}$ intercambiable $* \mathrm{~kg}^{-1}$ de suelo. La fertilización debe aplicarse en su totalidad al momento de la plantación y en forma localizada en la línea de plantación, $5 \mathrm{~cm}$. bajo el órgano subterráneo.

Mengel y Kirkby (2000), declaran que el potasio es el catión más importante en la fisiología de la planta, por estar contenido en el tejido vegetal, así como por las funciones fisiológicas y bioquímicas en las que interviene; así mismo promueve la translocación de fotosintatos.

En el medio científico no existen publicaciones relacionados con la fertilización de nitrógeno, fósforo y potasio en el I. amancaes, en este sentido es fundamental realizar investigaciones que determinen los requerimientos de dicha especie, a fin de lograr domesticarla.

El objetivo del presente estudio fue determinar el efecto de la fertilización en la producción de bulbos y flor de amancae, evaluado a través de las variables crecimiento (altura, diámetro de tallo, número de hojas y raíces) y rendimiento (número de flores, días a la floración, diámetro y peso de bulbo final), bajo condiciones de invernadero en el Programa de Investigación en Ornamentales de la universidad Nacional Agraria La Molina (UNALM), Lima, Perú.

\section{Materiales y métodos}

La presente investigación se desarrolló en el invernadero del Programa de Investigación en Ornamentales de la Universidad Nacional Agraria La Molina (UNALM), Lima, Perú. Localizada geográficamente a una latitud de $12^{\circ} 06^{\prime} \mathrm{S}$, longitud $76^{\circ} 57^{\prime} \mathrm{O}$ y altitud 243.7 m.s.n.m. Ubicado dentro de un desierto subtropical árido caluroso según el sistema modificado de Koppen (Garcia, 2004), con temperatura media anual de $18,5^{\circ} \mathrm{C}$, radiación anual de 186,5 cal-g* $\mathrm{cm}^{-1 *}$ día $^{-1}$, humedad relativa anual de $85 \%$ y precipitación anual acumulada de $6 \mathrm{~mm}$.

Se utilizaron bulbos de I. amancaes que fueron colectados del Santuario de Amancaes en Pachacamác, Lima, Perú. Los bulbos fueron colocados en casa malla, para que reposaran y se protejan del medio ambiente, por un periodo de 30 días. Luego de dicho período fueron llevados a cámara fría, a una temperatura de $10{ }^{\circ} \mathrm{C}$, por 21 días, con la finalidad de que los bulbos puedan salir de su estado de reposo. El día 52, los bulbos fueron retirados de la cámara fría y clasificados según su 
diámetro, en un rango de 3,79 a $4,01 \mathrm{~cm}$, con un peso promedio entre 29 a $33 \mathrm{~g}$, bulbos de tamaño mediano (Palacios, 2017). Se seleccionaron 216 bulbos, que fueron sembrados en bolsas de polietileno de 10 "x11" de ancho y largo respectivamente, Se utilizaron cinco litros de turba (Distichia muscoides) cortada y tamizada en una malla de celdas de $5 \mathrm{~cm}$. Las características químicas de la turba fueron: $6,31 \mathrm{de} \mathrm{pH}, 0,69 \mathrm{dS}^{*} \mathrm{~m}^{-1}$ de conductividad eléctrica, $53,13 \%$ de contenido de materia orgánica, 20,80 meq* $100 \mathrm{~g}^{-1}$ de capacidad de intercambio catiónico, 73,6\% de humedad en peso, $0,98 \% \mathrm{~N} ; 0,76 \% \mathrm{P}_{2} 0_{5}$ y $0,20 \% \mathrm{~K}_{2} \mathrm{O}$. $\mathrm{Su}$ uso como sustrato se debió a los resultados obtenidos por Vadillo y Suni (2006) quienes demostraron que es el más adecuado para el establecimiento de plántulas, porque mantiene la humedad y el $\mathrm{pH}$ estable lo que permite que las plántulas presenten un mayor vigor.

Las fuentes de fertilizantes utilizados fueron nitrato de amonio, superfosfato triple y cloruro de potasio. Los fertilizantes fosforados y potásicos se aplicaron al inicio de la siembra, mientras que el nitrógeno se fraccionó y aplicó en tres momentos, a los 15, 45 y 75 dias después de la siembra. Los tratamientos estuvieron constituidos por ocho diferentes dosis de fertilización: T1: 0-0-0, T2: 1200-0, T3: 0-50-0, T4: 0-0-160, T5: 120-50-0, T6: 120-0-160, T7: 0-50-160 y T8: 120-50-160. Cada tratamiento estuvo conformado por nueve bulbos.

Las variables evaluadas fueron: la altura de planta, fue obtenida por la medición en centímetros de la distancia entre el nivel del suelo y el extremo de la última hoja completamente desarrollada o extendida, con una regla milimetrada. El diámetro de tallo y el diámetro final del bulbo, fueron obtenidos por la medición en centímetros con un calibre. El número de hojas, raíces y flores, se obtuvieron a través del conteo por unidad. El peso final del bulbo, fue obtenido por el pesaje en una balanza electrónica y los días a la floración, fue obtenido en base al día de inicio de floración de la planta.

El diseño experimental que se utilizó fue el Diseño Completamente al Azar (DCA), con tres repeticiones. Las variables evaluadas fueron analizadas mediante el Análisis de Varianza (ANOVA), según Banzato y Kronka (1992), en la comparación de medias se usó la prueba de Duncan a un nivel de significancia de 0.05. Los análisis estadísticos se realizaron con un software libre.

\section{Resultados y discusión \\ Variables de crecimiento}

En la Tabla 1 se presenta el resultado de la comparación de medias por la prueba de Duncan, a un nivel de significancia de 0,05; para la característica altura de la planta, número de hojas por planta, número de raíces por planta y el diámetro de tallo de planta. En la cual se observa que el T8, resultó con valores significativamente inferiores a los obtenidos en el T1 en todas las variables de crecimiento evaluadas. Sin embargo, los resultados obtenidos del T1 guardan similitud estadística con el T3 y T4 para todas las variables a excepción de la variable altura en el tratamiento T4, que se observa es inferior estadísticamente. Estos resultados confirman la recomendación de Leroymerlin (2003), sobre la fertilización en plantas bulbosas, con abonos ricos en fósforo y potasio, pero bajos en contenidos de nitrógeno, para obtener plantas más vigorosas.

En otros estudios realizados por Agüero y Suni (1999); Agüero (2003); reportaron valores similares a los obtenidos en el T2 y T7 al evaluar al I. amancaes en la reserva Nacional de Lachay (Niño 97-98). Sin embargo, lo declarado por Soto (2015) solo difiere en altura de la planta con el T6. En relación al número de hojas, Agüero y Suni (1999); Agüero (2003) reportaron tres hojas, mientras que Suni et al. (2011) reportó cinco a nueve hojas, resultado superior al obtenido en los tratamientos del presente estudio. Estos resultados podrían deberse al contenido de nutrientes del bulbo. De acuerdo con Rees (1992) y Ortega-Blu et al. (2006) el bulbo, es un depósito natural de nutrientes, aún hasta la floración. Para Ibbett (1963), el bulbo es importante para hacer resistir a la planta en una estación poco favorable como es invierno y la prolongación de falta de agua. Además, De Hertogh y Le Nard (1993) señalaron que en las plantas bulbosas, como el amancae, los fotosintatos producidos por la parte aérea durante la temporada de crecimiento activo, son translocados a los órganos de reservas de tal forma que al momento de la plantación, las mayores acumulaciones de $\mathrm{N}, \mathrm{P}$ y $\mathrm{K}$ se encuentran en el bulbo (Ortega-Blu et al., 2006). Esas reservas son mantenidas durante el período de dormancia y son movilizadas cuando comienza el nuevo crecimiento. Di Benedetto (2007) reportó que al iniciar el brotamiento del bulbo, las reservas se desdoblan en moléculas simples, incluyendo la sacarosa, que se movilizan para sustentar el crecimiento del brote consumiéndose hasta el $75 \%$ de las reservas del bulbo madre. De esta manera, cualquier aporte externo de nutrientes en la temporada, tendría un efecto nulo o muy limitado en la misma temporada (Pinochet, 1999).

Tabla 1. Promedio de las variables de crecimiento de Ismene amancaes evaluadas bajo diferentes niveles de fertilización

\begin{tabular}{|c|c|c|c|c|}
\hline Tratamientos & $\begin{array}{l}\text { Altura de } \\
\text { la planta } \\
(\mathrm{cm})\end{array}$ & $\begin{array}{l}\text { Hojas por } \\
\text { planta } \\
\text { (undad) }\end{array}$ & $\begin{array}{l}\text { planta } \\
\text { (undad) }\end{array}$ & $\begin{array}{l}\text { ámetro de } \\
\text { de planta } \\
\text { (cm) }\end{array}$ \\
\hline T1 $0-0-0$ & $58,79 \mathrm{a}$ & $4,66 \mathrm{ab}$ & $3,87 \mathrm{a}$ & $1,25 \mathrm{a}$ \\
\hline T2 120-0-0 & $46,55 \mathrm{c}$ & $4,00 \mathrm{bc}$ & $2,64 \mathrm{c}$ & $1,09 \mathrm{~b}$ \\
\hline T3 $0-50-0$ & $61,81 \mathrm{a}$ & $4,66 \mathrm{ab}$ & $3,78 \mathrm{a}$ & $1,19 \mathrm{a}$ \\
\hline T4 0-0-160 & $52,03 \mathrm{~b}$ & $4,33 \mathrm{abc}$ & $3,74 \mathrm{a}$ & $1,25 \mathrm{a}$ \\
\hline T5 120-50-0 & $38,11 \mathrm{~d}$ & $3,66 \mathrm{bc}$ & $2,99 \mathrm{~b}$ & $1,06 \mathrm{bc}$ \\
\hline T6 120-0-160 & $24,49 \mathrm{e}$ & $2,33 \mathrm{~d}$ & $2,44 \mathrm{c}$ & $1,08 \mathrm{bc}$ \\
\hline T7 0-50-160 & $44,82 \mathrm{c}$ & $5,33 \mathrm{a}$ & $3,74 \mathrm{a}$ & $1,12 \mathrm{~b}$ \\
\hline T8 $120-50-160$ & $18,85 \mathrm{f}$ & $3,33 \mathrm{~cd}$ & $2,58 \mathrm{c}$ & $1,02 \mathrm{c}$ \\
\hline
\end{tabular}

*Medias seguidas de letras iguales, dentro de columnas, no difieren entre sí según prueba de Duncan, $\mathrm{p}=0,05$

\section{Variables de rendimiento}

En la Tabla 2 se presenta el resultado de la comparación 
de medias por la prueba estadística de Duncan, a un nivel de significancia de 0,05 ; para la característica número de flores por planta, días a la floración, diámetro de bulbo final, peso de bulbo final. En la cual se observa que el T1 mostró los valores significativamente más bajos de acuerdo a la prueba de Duncan.

Tabla 2. Promedio de las variables de rendimiento de Ismene amancaes evaluadas bajo diferentes niveles de fertilización

\begin{tabular}{llcccc}
\hline Tratamientos & $\begin{array}{c}\text { Número } \\
\text { de flores } \\
\text { por planta } \\
\text { (unidad) }\end{array}$ & $\begin{array}{c}\text { Días a la Diámetro de } \\
\text { floración } \\
\text { (días) }\end{array}$ & $\begin{array}{c}\text { Peso de } \\
\text { bulbo final } \\
\text { (cm) }\end{array}$ & $\begin{array}{c}\text { bulbo final } \\
\text { (g) }\end{array}$ \\
\hline T1 & $0-0-0$ & $1,00 \mathrm{c}$ & $117,3 \mathrm{e}$ & $3,88 \mathrm{~b}$ & $34,74 \mathrm{bcd}$ \\
T2 & $120-0-0$ & $1,00 \mathrm{c}$ & $133,3 \mathrm{c}$ & $3,90 \mathrm{~b}$ & $31,55 \mathrm{~d}$ \\
T3 & $0-50-0$ & $1,27 \mathrm{ab}$ & $118,6 \mathrm{e}$ & $3,98 \mathrm{~b}$ & $37,72 \mathrm{abc}$ \\
T4 & $0-0-160$ & $1,41 \mathrm{a}$ & $124,0 \mathrm{~d}$ & $4,20 \mathrm{a}$ & $40,89 \mathrm{a}$ \\
T5 & $120-50-0$ & $1,00 \mathrm{c}$ & $137,0 \mathrm{c}$ & $3,88 \mathrm{~b}$ & $31,61 \mathrm{~d}$ \\
T6 & $120-0-160$ & $1,00 \mathrm{c}$ & $138,0 \mathrm{c}$ & $3,98 \mathrm{~b}$ & $32,61 \mathrm{dc}$ \\
T7 & $0-50-160$ & $1,41 \mathrm{a}$ & $144,0 \mathrm{~b}$ & $3,96 \mathrm{~b}$ & $38,33 \mathrm{ab}$ \\
T8 & $120-50-160$ & $1,13 \mathrm{bc}$ & $149,3 \mathrm{a}$ & $3,89 \mathrm{~b}$ & $31,05 \mathrm{~d}$ \\
\hline
\end{tabular}

*Medias seguidas de letras iguales, dentro de columnas, no difieren entre sí según prueba de Duncan, $\mathrm{p}=0,05$

Según Brito (2006) en el proceso de floración están involucradas cinco etapas sucesivas: inducción, iniciación, organogénesis, maduración y antesis, siendo la disponibilidad de nutrientes uno de los factores que controlan dicho proceso (Halevy, 1990). Los resultados obtenidos muestran una tendencia al aumento en los días de floración, con la fertilización. El T1 y T3 fueron los primeros que comenzaron con la floración, estos resultados pueden deberse al estrés producto de la deficiencia de nutrientes (Levy y Dean 1998), mientras que el T8 fue el que demoró más días en emitir botones florales. Este efecto se puede deber a la influencia de la salinidad (Mengel y Kirkby, 2000) por la aplicación de los fertilizantes, principalmente el cloruro de potasio y el nitrato de amonio. De acuerdo con Salisbury y Ross (1985); Martinez et al. (1987), la presencia de estas sales disueltas en la solución suelo exigen un mayor gasto de energía de origen metabólico para absorber el agua a través de su sistema radicular, conllevando a un detrimento de esta energía en otras funciones de la planta, que también la requiere para su crecimiento, la floración, entre otros. También se observó que los tratamientos que contenían nitrógeno fueron los que más días tardaron en entrar en floración, a excepción del T8. Esto pudo deberse a la aplicación de una elevada dosis de nitrógeno que ocasionó retardo en la floración y formación de semillas (Azcón-Bieto y Talón 2000).

El T4 superó significativamente a los otros tratamientos en relación al número de flores por planta, diámetro de bulbo y peso final de bulbo; excepto al T7, con relación al número de flores y peso de bulbo. Estos resultados se pueden deber al aporte de nutrientes que contenía el sustrato (musgo) dentro de su composición, que fue $53,13 \%$ de materia orgánica, $0,98 \%$ de $\mathrm{N}$ y $0,76 \%$ de $\mathrm{P}_{2} 0_{5}$, estos minerales pudieron liberarse durante el proceso de humificación, pasando a estar disponible el $\mathrm{N}$, parte de $\mathrm{P}$ y con el aporte externo de la fuente potásica, se logró tener un mayor diámetro y peso final del bulbo de amancae en el T4. Así mismo, la aplicación de la dosis de $\mathrm{N}$ aparentemente fue en exceso, por lo que los tratamientos que tuvieron dentro de su composición $\mathrm{N}$, son los que menor peso de bulbo final presentaron. Estos resultados concuerdan con la recomendación de Leroymerlin (2003) sobre a la fertilización con nutrientes ricos en fósforo y potasio, pero deficientes en nitrógeno en plantas bulbosas. Contrario a lo reportado por Pinochet (1999) quien indica que el bulbo no cubre la demanda total de $\mathrm{N}$ para producir flores, siendo necesario fertilizar con $\mathrm{N}$ para compensar el déficit en la producción de flores y follaje. Sin embargo, Suni et al. (2011) y Palacios (2017) reportaron que el número de flores por planta depende del tamaño del bulbo, debido que esta es una estructura de reserva (Rees, 1992). Pero Ortega-Blu et al. (2006) al realizar un estudio en Lilium spp. determinaron que los nutrientes aportados por el bulbo no es suficiente para completar el ciclo de cultivo para flor de corte, siendo una práctica necesaria fertilizar, en la producción comercial de flores.

El mayor peso del bulbo obtenido en los T4 y T7 se pueden deber a la no fertilización con 120 de $\mathrm{N}$, que guarda relación con la maduración del follaje activo de las plantas de amancae. Según lo reportado por Di Benedetto (2007); Pinochet, (1999); Bañon et al. (1993) a mayor cantidad de fotoasimilados producidos por la parte aérea, durante la estación de activo crecimiento los nutrientes, son translocados a los órganos de reserva hasta la muerte de dicha biomasa. Así mismo, Mengel y Kirkby (2000) declararon que el ión potasio dentro de la planta promueve la translocación de fotosintatos recién sintetizados y reservas almacenadas. Esta translocación y acumulación de reservas explicaría el mayor crecimiento del bulbo lo cual repercutirá en una mejor floración en la siguiente campaña (Pinochet, 1999; Ortega-Blu et al., 2006).

\section{Conclusiones}

Este estudio demuestra que las variables de crecimiento (altura, diámetro de tallo, número de hojas y raíces) no son favorecidas significativamente por la aplicación de fertilizantes edáficos (NPK). Sin embargo, fertilizar con el $\mathrm{T}_{4}$ se obtiene un mejor resultados para la variable de rendimiento (número de flores, peso y diámetro del bulbo del $I$. amancaes) y con el T8 se prolonga el inicio de la floración a 149,3 días. Se recomienda investigar el balance nutricional y la oferta de nutriente para obtener óptimos resultados en las variables estudiadas, lo que permitirá domesticar a esta especie de valor ornamental.

\section{Literatura citada}

Agüero, S. 2002. Efecto de la humedad edáfica en el desarrollo y propagación de Ismene Amancaes $R \& P$. Herbert "amancaes" (Amaryllidaceae) en condiciones 
“in situ” $y$ "ex situ” (Tesis para optar el Título Profesional de Biólogo con Mención en Botánica). Universidad Nacional Mayor de San Marcos, Lima, Perú. 94 p.

Agüero, S. 2003. Influencia de la humedad edáfica en el ciclo biológico y propagación de Ismene amancaes (R. \& P.) Herbert “amancaes"(Amaryllidaceae). Resúmenes: I-Congreso Internacional de Científicos Peruanos 2003 (I-CICP 2003).

Agüero, S. y Suni, M. 1999. Influencia del evento "El Niño 1997-98" en el desarrollo de Ismene amancaes (Amaryllidaceae, Liliopsidae). El Niño 1997-98 y su Impacto sobre los Ecosistemas Marino y Terrestre. Revista Peruana Biología. Vol. Extraordinario: 118124.

Azcón-Bieto, J. y Talón, M. 2000. Fisiología Vegetal. Ediciones Universidad de Barcelona. Edit. McGrawHill Interamericana. 522 p.

Bañon, S.; Cifuentes, D.; Fernández, J. y González, A. 1993. Gerbera, Lilium, Tulipan y Rosa. Eds MundiPrensa. Madrid, ES. 250 p.

Banzato, D.A. y Kronka, S.N. 1992. Experimentação Agrícola. Jaboticabal Funep, SP. Brasil. 245p.

Brito, C. 2006. Morphological analysis of tropical bulbs and environmental effects on flowering and bulb development of Habranthus and Zephyranthes spp. (Tesis de Maestría). Universidad de Florida. Florida, Estados Unidos.

De Hertogh, A. and Le Nard, M. 1993. Botanical aspects of flower bulbs. En: De Hertogh, A. and M. Le Nard. (Eds). The physiology of flower bulbs. HO. 29-43.

Di Benedetto, A. 2007. Producción de especies ornamentales en maceta, un enfoque ecofisiológico. Orientación Gráfica Editora. ARG. 196 p.

Garcia, E. 2004 Modificaciones al sistema de clasificación climática de Köppen. Quinta edición, Instituto de Geografía -UNAM. Recuperado de: Mexico http:// www.igeograf.unam.mx/sigg/utilidades/docs/pdfs/ publicaciones/geo_siglo $21 /$ serie lib/modific al sis. pdf

Halevy, A. H. 1990. Recent advances in control of flowering and growth habit of geophythes. Acta Hort., 266:35-42.

Ibbett, C. 1963. Producción comercial de bulbos. Manual de técnicas agropecuarias. Edit. ACRIBIA. Zaragoza. $155 \mathrm{p}$.

La República. 2017. La fiesta de la Flor de Amancaes. Recuperado de: http://larepublica.pe/perusorprendente/872484-la-fiesta-de-la-flor-de-amancaesvideo.

Leroymerlin, 2003. Cultivar plantas bulbosas. Recuperado de:: $\quad$ http://agro.unc.edu.ar/ biblio/Como $\% 20$ cultivar $\% 20$ plantas $\% 20$ de $\% 20$ bulbo.pdf

León, R. 2002. Evaluación de la aplicación de fertilización nitrogenada sobre la producción deflores y bulbificación de Leucocoryne purpurea (Taller de licenciatura Agr.
Quillota). Universidad Catolica de Valparaiso, Chile.

León, B.; Saguestegui, A.; Sánchez, I.; Zapata, M.; Meerow, A. \& A. Cano, A. 2006. Amaryllidaceae endémica del Perú. En: El libro rojo de las plantas endémicas del Perú. León B. et al. (Eds). Rev.Per.Biol. Número especial 13 (2): 690-697

Levy, Y.; and Dean, C. 1998. The transitium to flowering. Plant cell, 10: 1973-1990.

López-Mtz, J.; Díaz, A.; Martínez, E. y Valdez, R. 2001. Abonos orgánicos y su efecto en propiedades físicas y químicas del suelo y rendimiento en maíz. Terra Latinoamericana 19 (4): 293-299.

Magalhães, J.R. 1993. Nutrição e adubação da cebola. In: Simpósio sobre Nutrição e Adubação de Hortaliças, 1990, Jaboticabal, SP. Anais. Piracicaba: Potafos, p.381-399.

Martínez, A.; Aragües, R. and Royo, A. 1987. Salt tolerance of barley (Hordeum vulgare L.) cultivars at the germination stage: Analysis of the responses functions. Plant and Soil 104: 53-56.

Mengel, K. y Kirkby, E. 2000. Principios de nutrición vegetal. Traducido por: R. Melgar y M. Gallud. Ed. International Potash Institute. $692 \mathrm{p}$.

Mollenhauer, C. 2002. Determinación de los parámetros necesarios para la demanda potásica del cultivo del tulipán (Tulipa sp.) (Tesis presentada como parte de los requisitos para optar al grado de Licenciado en Agronomía). Universidad Austral de Chile, Chile. Recuperado de: http://cybertesis.uach.cl/tesis/ uach/2002/fam726d/doc/fam726d.pdf

Ortega-Blu, R.; Correa-Benguria, M.; Olate-Muñoz y E. 2006. Determinación de las curvas de acumulación de nutrientes de tres cultivares de Lilium spp. para flor de corte. Agrociencia 40: 77-88. Recuperado de: http:// www.redalyc.org/html/302/30240108/.

Palacios, J. 2017. Momento de riego y tamaño de bulbo en el rebrote y floración del Amancay (Ismene amancaes. Ruiz \& Pavón). Anales científicos 78 (1): 1-10. doi: http://dx.doi.org/10.21704/ac.v78i1.873

Pascual, E. 2007. Propagación in vitro de Ismene amancaes (R.\&P.) Herbert "Amancay" (Amaryllidaceae) (Tesis para optar el Título Profesional de Biólogo con Mención en Biología Celular y Genética). Universidad Nacional Mayor de San Marcos, Lima, Perú. Recuperado de: http://cybertesis.unmsm.edu.pe/xmlui/bitstream/ handle/cybertesis/878/Pascual_me.pdf? sequence $=1$

Pinochet, D. 1999. Fertilización de plantas bulbosas. En: Seemann, P. y N. Andrade, (eds.). Cultivo y manejo de plantas bulbosas ornamentales. Universidad Austral de Chile, Facultad de Ciencias Agrarias. CH. 123-137

Rees, A.R. 1992. Ornamental bulbs, corms and tubers. Wallingford, CAB International. 220 p.

Salisbury, F. B. and Ross, C. W. 1985. Plant Physiology, Third ed. Wandsworth Publishing Co. Inc. Belmont, California. 540 p. 
Sánchez, C. E. 2006. Caracterización del estado nutricional y fisiológico en plantas de judía (Phaseolus vulgaris $L$. cv. Strike) sometidas a un estrés por nitrógeno. Tesis doctoral. Universidad de Granada. ESP. 206 p.

Servicio Nacional Forestal y de Fauna Silvestre (SERFOR). 2015. Guía de Flora de las Lomas de Lima Book March 2015 with 10,183 Reads, DOI 10.13140/ RG.2.1.4431.0564, Edition 1a Edición, ISBN978-61246908-0-8. Recuperado de: https://www.researchgate. net/publication/274374014_Guia_de_Flora_de_las Lomas de Lima

Soto, M. y Leiva, M. 2015. Estudio exomorfológico y fitoquímico de los bulbos de dos especies endémicas del Perú de la familia Amaryllidaceae. Arnaldoa 22 (1): 269 - 288. Recuperado de: http://journal.upao.edu.pe/ Arnaldoa/article/view/194/182

Suni, M.; Pascual, E. y Jara, E. 2011. Desarrollo reproductivo del "amancay" Ismene amancaes (Amaryllidaceae) en su ambiente natural. Rev. peru biol. 18(3): 293 - 297.

Ulloa, C.; Zarucchi, J. L. y León, B. 2004. Diez años de adiciones a la Flora del Perú: 1993-2003. Arnaldoa Edic. Espec., Nov. 2004: 109-156.

Vadillo, G. y Suni, M. 2006. Evaluación de sustratos para el establecimiento en laboratorio de plántulas de Puya raimondii Harms (Bromeliaceae). Rev. peru. biol. 13(1): 139 - 141. Recuperado de: http://www.scielo.

org.pe/pdf/rpb/v13n1/a14v13n01.pdf 